\title{
¿POR QUÉ EL ADULTO MAYOR CON HIPERTENSIÓN ARTERIAL REQUIERE DE UN CUIDADO ESPECIFICO?
}

\begin{abstract}
WHY ELDERLY ADULTS WITH HIGH BLOOD PRESSURE REQUIRES SPECIFIC CARE?
\end{abstract}

Recibido enero 2017 Aceptado febrero 2017

\section{Autores:}

Esparza Méndez RM

Autora del artículo.

Estudiante de la Maestría en Ciencias de Enfermería, Universidad de Guanajuato,

Campus Celaya Salvatierra

Santos Degollado \#195. Zapotlán El Grande, Ciudad Guzmán, Jallisco. C.P. 49000. Teléfono: 045 (317) 1019089 Correo electrónico: bldrosae@hotmail.com

Jiménez González MJ

Maestra en Ciencias de Enfermería. Docente Universidad de Guanajuato,

Campus Celaya Salvatierra

Departamento de Enfermería Clínica. Av. Ing. Javier Barros Sierra No. 201 Esq. Av. Baja California Ejido Santa María del Refugio. Celaya, Guanajuato. CP 38140. Teléfono (467) 5985922.

Moreno Pérez NE

Doctora en Enfermería. Docente

Universidad de Guanajuato,

Campus Celaya-Salvatierra

Departamento de Enfermería Clínica. Av. Ing. Javier Barros Sierra No. 201 Esq. Av. Baja California Ejido Santa María del Refugio. Celaya, Guanajuato. CP 38740. Teléfono (467) 5985922.

Palabras clave: hipertensión arterial, funciones cognitivas, adulto mayor

Keywords: high blood pressure, cognitive functions, elderly. 


\section{RESUMEN}

Introducción: los grupos de enfermedades vinculadas a la edad aumentaron notablemente en los últimos años, la exposición a problemas de salud a lo largo de toda una vida impacta en el hecho de las personas llegan a la tercera edad con enfermedades crónicas y una salud deteriorada. Las consecuencias fisiopatológicas de la hipertensión arterial en el cerebro suelen afectar a las áreas subcorticales prefrontales y producir déficit en la abstracción, formulación de objetivos y funciones ejecutivas. Los componentes de las funciones cognitivas que se relacionaron con alteraciones de la presión arterial en los estudios analizados fue la memoria, las funciones ejecutivas, el lenguaje y la atención. En particular, la presencia de alteraciones o daño en las funciones ejecutivas no solo genera disminución en la capacidad funcional del individuo, sino que además ocasionan ciertos cambios en la dinámica familiar de la persona afectada. Incluso, la persona se vuelve más susceptible a presentar cierto grado de disfunción que puede cambiar su calidad de vida de forma irreversible, provocando disminución en la funcionalidad que más tarde se puede traducir en incapacidad física y cognitiva.

Palabras clave: hipertensión arterial, funciones cognitivas, adulto mayor.
ABSTRACT

Introduction: age-related disease groups have increased significantly in recent years, exposure to health problems over a lifetime impacts on the fact that people reach the elderly with chronic diseases and deteriorated health. The pathophysiological consequences of arterial hypertension in the brain often affect prefrontal subcortical areas and produce deficit in abstraction, goal setting, and executive functions. The components of the cognitive functions that were related to alterations of the blood pressure in the analyzed studies were the memory, the executive functions, the language and the attention. In particular, the presence of alterations or damage in executive functions not only generates a decrease in the functional capacity of the individual, but also causes certain changes in the family dynamics of the affected person. Even the person becomes more susceptible to some degree of dysfunction that can change their quality of life irreversibly, causing a decrease in functionality that can later translate into physical and cognitive disability.

Keywords: high blood pressure, cognitive functions, elderly.

\section{4}




\section{INTRODUCCIÓN}

os grupos de enfermedades relacionadas con la edad aumentaron notablemente, la exposición a problemas de salud a lo largo de la vida impacta de manera importante cuando las personas llegan a la tercera edad, principalmente a causa de enfermedades crónicas que generan una mala calidad de vida y por ende la salud deteriorada.

El envejecimiento, no sólo se manifiesta en los indicadores demográficos, también se hacen presentes en la individualidad, es decir, los cambios fisiológicos del envejecimiento predisponen a procesos patológicos que se expresan como enfermedades dependientes de este, ocasionando declinaciones a nivel musculo-esquelético, cardiaco, elasticidad de la piel y de las grandes arterias, que sumado a la presencia de ciertos factores contribuirán en la aparición de la cronicidad?

Como es sabido las enfermedades no transmisibles (ENT) representan uno de los mayores desafíos a nivel mundial, debido a que representan la principal causa de muerte, habiendo ocasionado el 68\% de las defunciones registradas en el 2012.

En particular la hipertensión arterial (HTA), es un problema actual de salud pública, el cual ocupa un lugar importante dentro de las principales enfermedades no transmisibles, por lo que el impacto que genera trasciende a los aspectos fisiológicos, cognitivos y conductuales que repercuten más tarde en la calidad de vida de la persona que padece dicha enfermedad.

A fin de retrasar el impacto que ocasiona la HTA, se han establecido metas las cuales ponen de relieve la importancia de llevar a cabo un plan de manejo, el cual incluye el establecimiento de metas de tratamiento hipertensivo en el manejo no farmacológico, el tratamiento farmacológico, la educación del paciente, y la vigilancia de complicaciones. ${ }^{3}$
Sin embargo, mientras que el objetivo del tratamiento antihipertensivo en el adulto se plantea en términos de disminución de la morbimortalidad cardiovascular y renal, el objetivo primordial para el adulto mayor (AM) va encaminado a la prevención del evento cerebrovascular, mantener la expectativa de vida libre de discapacidad y la maximización de la función. ${ }^{4}$

Cabe señalar que la meta principal a tratar en la hipertensión, es la reducción del riesgo cardiovascular global, y las cifras de presión arterial (PA) se adecuaran a las características de cada persona. ${ }^{5}$ Los expertos del Joint National Committee (JNC) en su octava reunión hacen la recomendación que en el caso de los adultos de 60 años o más años, la meta de la presión arterial sistólica (PAS) deberá ser menor de 150 mm Hg y en la presión arterial diastólica (PAD) menor de 90 mm Hg sin ser inferior a 65 mm Hg dado el riesgo de hipoperfusión coronaria?

En el caso de los AM, el tratamiento será iniciado con manejo no farmacológico, dirigido a la reducción del consumo de sodio, el control de peso, aumento de la actividad física, la disminución del consumo de alcohol y la restricción de hábito del tabaquismo. ${ }^{5}$ Con respecto al tratamiento farmacológico deberá tenerse vigilancia específica en la comorbilidad y la polifarmacia, particularmente frecuente. ${ }^{8}$

Por lo tanto, se deberá tener una mayor vigilancia al cumplimiento del plan terapéutico considerando los aspectos farmacológicos y conductuales del AM, ya que el descontrol de la enfermedad puede ocasionar pérdida de la capacidad de autorregulación del flujo sanguíneo cerebral. ${ }^{9}$

Esto sin duda, pone en mayor vulnerabilidad a los adultos mayores, debido a que la HTA tiene efectos importantes en la regulación de la circulación cerebral, que afectan a la estructura y función del cerebro mediante la reducción de las reservas vasculares, la isquémica, patología vascular adicional, manifestada por interrupciones de la función de barrera de la sangre-cerebro, 
reducción de la neuroconectividad funcional, remodelación vascular, inflamación sistémica, disfunción endotelial y/o arterioesclerosis en las grandes arterias cerebrales y cervicocerebrales, entre muchas otras condiciones que promueven la enfermedad cerebrovascular. ${ }^{10}$

Los cambios cerebrovasculares derivados del descontrol de la HTA, se asocian con alteraciones vasculares, ocasionando alteraciones en la materia blanca, dichos cambios pueden contribuir en la aparición de deterioro cognitivo. ${ }^{11}$

Además, existe evidencia que relaciona la HTA con las principales entidades causantes, ya sea a través del ictus sintomático o mediante las consecuencias fisiopatológicas que ocasiona la rigidez en las pequeñas arterias cerebrales, provocando lesión de la sustancia blanca y daño isquémico, ocasionando no solo ataque vasculo-encefálico, sino también el de enfermedades neurocognitivas tales como el deterioro cognitivo leve y la demencia. ${ }^{12}$

Se debe tener presente que el deterioro cognitivo en sus diferentes componentes puede tener origen a nivel vascular, principalmente a través de la enfermedad vascular cerebral y la enfermedad de pequeños vasos, que provocan alteraciones cerebrales que afectan la función e independencia de los adultos mayores. ${ }^{13}$

Estos déficits cognitivos relacionados con la HTA suelen ser sutiles y afectan múltiples dominios neuropsicológicos, entre ellos el aprendizaje, la memoria, la atención, el razonamiento abstracto, la flexibilidad mental y las habilidades psicomotrices y visuoespaciales, produciendo a su vez déficit en la abstracción, formulación de objetivos dentro del componente de las funciones ejecutivas. ${ }^{12-14}$

Incluso en los últimos años, las directrices en la investigación se han dirigido a determinar la relación que existe entre la hipertensión arterial y las funciones cognitivas, lo cual sin duda, ha permitido aclarar ciertas particularidades sobre la asociación de estas variables, de forma específica en el envejecimiento. Al analizar la asociación entre las funciones cognitivas y las alteraciones de la presión arterial se encontró que la memoria, las funciones ejecutivas, el lenguaje y la atención se reportaron alteradas con mayor frecuencia en los estudios (ver cuadro 1).

Cabe mencionar que estas funciones están estrechamente relacionadas con el lóbulo frontal, el cual se ubica en la porción anterior a la corteza y se sitúan por delante de la cisura central y por encima de la cisura lateral, ${ }^{26}$ se encuentra conformado por tres áreas: la corteza motora, premotora y prefrontal. Siendo en particular la corteza prefrontal (CPF), una de las áreas más complejas debido a que permite integrar información proveniente de señales motivacionales, emocionales, mnésicas, somatosensoriales y unificarlas para guiar nuestra conducta hacia la resolución de situaciones novedosas. ${ }^{27}$

Incluso, cabe mencionar que la CPF a menudo se clasifica como "la corteza de asociación multimodal", esto se debe principalmente al procesamiento de la información de diversas modalidades sensoriales que se integran de manera precisa para formar las construcciones fisiológicas de la memoria, la percepción y diversos procesos cognitivos. ${ }^{28}$

Por lo tanto, aun cuando el lóbulo frontal no está directamente implicado en el almacenamiento de la información per se, interviene en los procesos estratégicos de codificación, recuperación, monitorización y verificación, ${ }^{29}$ de alguna manera esta estructura también ejerce el papel supervisor e «inteligente» de trabajar con la memoria". ${ }^{30}$

Otra de las funciones cognitivas más complejas y evolucionadas del ser humano que se encuentra en el lóbulo frontal: es el sistema supramodal de procesamiento múltiple, mejor conocido como "funciones ejecutivas" (FE). ${ }^{37}$ El término "funciones ejecutivas", es definido por Muriel Lezak como: "las capacidades mentales esenciales para llevar a cabo una conducta eficaz, creativa y aceptada socialmente". 32

\footnotetext{
46 MN Why Enf Neurol Vol. 16. No. 1 enero - abril 2017
} 
Según Fuster, la principal capacidad de las FE es la habilidad temporal de organizar el comportamiento, el lenguaje y el razonamiento. ${ }^{33}$ Este componente se encarga también del control de procesos como; la inteligencia, la creatividad, la ejecución de actividades complejas, la toma de decisiones o el juicio ético y moral. ${ }^{32}$ Sin embargo, existe una relación reciproca con otras funciones cerebrales en una doble dirección.

En investigaciones realizadas recientemente, se encontró que la zona ventromedial prefrontal, está relacionada con la capacidad de toma de decisiones, planificación y razonamiento, los cuales forman componentes de las FE ${ }^{34}$. Estas habilidades cognitivas, permiten a la persona resolver problemas tanto internos (área afectiva, cognitiva y emotiva), como externos (interacción con ambiente, social y cultural). La información que recibe el cerebro a través de estos componentes es clave para llegar a una adecuada solución de problemas. ${ }^{31}$

Aunado a lo mencionado, es preciso considerar los cambios que ocurren en el desempeño cognitivo asociados al envejecimiento fisiológico, los cuales se correlacionan con múltiples cambios morfológicos y funcionales en el sistema nervioso central. ${ }^{35}$ En particular, el envejecimiento cerebral, es un proceso complejo que suele afectar de modo diferente a ciertas partes del cerebro, siendo el área cortical una de las más afectadas por el proceso degenerativo de la corteza, trayendo como resultado la atrofia y muerte neuronal. ${ }^{36}$

De forma particular, se resaltan los cambios que se generan durante el envejecimiento normal o patológico en el lóbulo frontal, el cual sufre importantes modificaciones anatómicas relacionadas con la reduccióndelnúmerodeneuronasysufuncionamiento; cabe señalar que estas modificaciones cerebrales surgen con anterioridad en el lóbulo frontal que en otras regiones cerebrales. ${ }^{37}$

Inclusosehaidentificadoqueduranteelenvejecimiento normal ocurren dos procesos muy importantes, el primero tiene que ver con la disminución significativa del número de células estaminales neuronales, la reducción en la producción de neuronas. El segundo proceso se relaciona con la microglía; una de las principales líneas de defensa del sistema nervioso central que apoya a las funciones cerebrales. ${ }^{38}$

Sin embargo, al ocurrir algún daño o enfermedad, en este caso provocado por la HTA, ocasiona el deterioro de este tipo de células, las cuales se relacionan con el desarrollo de procesos neurodegenerativos. ${ }^{38}$ Esto sin duda, es de gran relevancia biomédica puesto que además de ser muy frecuente, dependiendo de su magnitud, pueden ser importantes determinantes de discapacidad. ${ }^{35}$

Las implicaciones de las funciones ejecutivas en el autocuidado del adulto mayor tienen que ver principalmentecon los procesos neurodegenerativos, ya sea por el envejecimiento normal o patológico.

Al respecto, es importante mencionar que dicho proceso se ha relacionado con ciertas alteraciones en diferentes componentes cognitivos, dentro de los cuales se destacan "las funciones ejecutivas", cuya función permite que la persona pueda ser capaz de llevar a cabo una vida independiente y socialmente adaptada, esto se debe principalmente a que los procesos ejecutivos están ligados con la realización de actividades encaminadas al autocuidado, la adaptación con el entorno y en menor o mayor medida al desarrollo del rol social en el individuo. ${ }^{39}$

Incluso algunos autores refieren que, la presencia de alteraciones o daños en el funcionamiento ejecutivo están relacionados con secuelas derivadas de lesiones (traumatismos craneoencefálicos, accidentes vasculares cerebrales, tumores y enfermedades infecciosas) que ocasionan algún tipo de discapacidad cognitiva o funcional. ${ }^{40}$

En particular, los accidentes vasculares cerebrales, son una de las principales causas de incapacidad en adultos mayores, cuya predisposición se relaciona con la progresión del envejecimiento y los cambios negativos en el estilo de vida, conduciendo a la aparición de enfermedades no transmisibles (HTA, DM, dislipidemías). ${ }^{40}$

Enf Neurol Vol. 16. No. 1 enero - abril 2017 
Cabe señalar que las alteraciones o daño en las FE no solo generan la disminución en la capacidad funcional del individuo, sino que además, condicionan alteraciones la dinámica familiar.

Debido a ello, es importante la integración y trabajo conjunto multidisciplinar el cual contribuya con:

- La valoración oportuna del funcionamiento cognitivo en el adulto mayor;

- La generación de estrategias innovadoras que favorezcan el cuidado integral e individualizado del adulto mayor con hipertensión arterial;

\section{REFERENCIAS BIBLIOGRÁFICAS}

1. López R, Jaureguí J. Fisiología del envejecimiento. 2 ed. Colombia: Celsus; 2012

2. Organización Mundial de la Salud. Informe sobre la situación mundia de las enfermedades no transmisibles 2014: Resumen de orientación. OMS; 2014. $\mathrm{WHO} / \mathrm{NMH} / \mathrm{NVI} / 15.1$.

3. Hernández M, Lezana M, Barriguete-Meléndez J, Mancha C, et al. Guía de tratamiento farmacológico y control de la Hipertensión Arterial Sistémica. Rev Cardio. (Mex). 2011; 22 (1):6.

4. Herrera J, González-Miranda M, R. Robles N, et al. La hipertensión arterial en los pacientes octogenarios. Reflexiones sobre los objetivos, el tratamiento y sus consecuencias. 2017: 4 (3):18-28.

5. Valenzuela-Flores AA et al. Guía de práctica clínica: Recomendaciones de la guía de práctica clínica de hipertensión arterial en el primer nivel de atención hipertensión arterial. Rev Med Inst Mex Seguro Soc. (Mex). 2016; 54 (2): 249-60.

6. James PA, Oparil S, Carter BL, et al. Evidence-Based Guideline for the Management of High Blood Pressure in Adults Report From the Panel Members Appointed to the Eighth Joint National Committee (JNC 8). JAMA. 2014; 311 (5): 507-520.

7. Diagnóstico y tratamiento de la hipertensión arterial en el primer nivel de atención. México: Instituto Mexicano del Seguro Social; 08/07/2014

8. Hanon $O$. Hipertensión arterial en el anciano. EMC -Tratado de Medicina. 2014; $18(1): 7-6$.

9. Román $G$. Pascual B. Demencia vascular y deterioro cognitivo de origen vascular. Rev Neuro Neuropsi y Neuroc (Col). 2012; 12 ( $N^{\circ}$ 7): 203-78.
- El desarrollo de actividades que promuevan el autocuidado y fortalezcan la autosuficiencia.

Como lo expone el documento el tratamiento de la hipertensión arterial, requiere de la vigilancia y control de innumerables factores, sin embargo, en el caso de los adultos mayores, los cuidados deben ser mucho más específicos dadas sus características fisiológicas, cognitivas y conductuales.

\section{Agradecimientos}

Agradezco a CONACyT por el apoyo económico que me otorgó para cubrir mis gastos de manutención en mis estudios de posgrado.
10. ladecola C. Hypertension and Dementia. Hypertension. 2014; 64 (1):3-5 doi: 10.1767/hypertensionaha. 174.03040

11. Gasecki D, Kwarciany M, Nyka W, Narkiewicz K. Hypertension, brain damage and cognitive decline. Current Hypertension Reports. 2013; 15 (6):547-58

12. De Cerchio E, Parras J, Reyes ML, Vicario A. Deterioro cognitivo y rigidez arterial. Rev Fed Arg Cardiol. (Arg) 2015; 44 (Supl.7 HTA): 28-32.

13. Muñoz-Pérez MJ, Espinosa-Villaseñor D. Deterioro cognitivo y demencia de origen vascular. Rev Mex Neuroci (Mex). 2016; 17 (6): 85-96

14. Sabayan B, Wijsman L, Foster-Dingley J, Stoott D, Ford I, Buckeley B, et al Association of visit-to-visit variability in blood pressure with cognitive function in old age: prospective cohort study. BMJ. 2013; 347: 2-17.

15. Bucur B, Madden D. Effects of Adult Age and Blood Pressure on Executive Function and Speed of Processing. Exp Aging Res .2010; 36 (2):7-12

16. Fischer AL, Bernstein DM, Thornton WL. Vascular health modifies theory of mind performance in older adults. J Gerontol. 2014; 69 (2):219-27.

17. Goldstein F, Levey A, Steenland N. High Blood Pressure and Cognitive Decline in Mild Cognitive Impairment. J Am Geriatr Soc. 2013; 61 (1):67-73.

18. Hsu-ko, et al. Effect of Blood Pressure on Cognitive Functions in Elderly Persons. J Gerontol A Biol Sci Med Sc. 2004; 59 (11):1191-4.

19. Muller M, Sigurdsson S, Kjartansson O. Joint effect of mid and late life blood pressure on the brain: The AGES-Reykjavik Study. Neurology. 2014. 82(24):2187-95

48 MMN Why Enf Neurol Vol. 16. No. 1 enero - abril 2017 
20. Matoso J, Santos B, Moreira I, Lourenço R, Correia M. Elderly hypertensives show decreased cognitive performance compared with elderly normotensives. Ara Bras Cardiol (Brasil). 2013; 100 (5):444-51.

21. McDade E, Sun Z, Lee C-W, Snitz B, Hughes T, Chang C-CH, et al. The association between pulse pressure change and cognition in late life: Age and where you start matters. Alzheimer's \& Dementia: Diagnosis, Assessment \& Disease Monitoring. 2016; 4:56-66

22. Elias M, Dore G, Davey A, Robbins M, Elias P. From blood pressure to physical disability: The role of cognition. Hypertension. (Philadelphia). 2010; $55(6): 7360-5$

23. Nation DA, Wierenga CE, Delano-Wood L, Jak AJ, Delis DC, Salmon DP, et al. Elevated pulse pressure is associated with age-related decline in language ability. Journal of the International Neuropsychological Society. 2010; 16 (5):933-8

24. Spinelli C, De Caro MF, Schirosi G, Mezzapesa D, De Benedittis L. Chiapparino C, et al. Impaired cognitive executive dysfunction in adult treated hypertensives with a confirmed diagnosis of poorly controlled blood pressure. Int J Med Sci. 2014; 11 (8): 771-8.

25. Waldstein, Shari R, Brown J, Maier K, Katzel L. Diagnosis of hypertension and high blood pressure levels negatively affect cognitive function in older adults. Ann Behav Med. 2005: 29 (3):174-80

26. Lozano-Gutiérrez A, Ostrosky-Solis F. Desarrollo de las funciones ejecutivas y de la corteza prefrontal. Rev Neuropsi, Neuropsi y Neuroc. (Mex). 2011; 11 (№7): 159-172.

27. Bruna O, Roig T, Puyuelo M, Junqué C, Ruano Á. Rehabilitación neuropsicológica: Intervención y práctica clínica. España; Elservier: 2011.

28. Ardila A, Ostrosky F. Guía para el Diagnóstico Neuropsicológico. México: 2012

29. Clemente Y, García-Sevilla J, Méndez I. Memoria, funciones ejecutivas y deterioro cognitivo en población anciana. European j investiga. 2015; 5 (2): 17 .
30. Cabeza R, Moscovitch M. Memory Systems, Processing Modes, and Components: Functional Neuroimaging Evidence. Perspectives on psychological science: a journal of the Association for Psychologica Science. 2013; 8 (1):49-55.

31. Tirapu-Ustárroz J, Garcia-Molina A, Rios-lago M, Pelegrin-Valero C Funciones ejecutivas. Neuropsicología de la corteza prefrontal y las funciones ejecutivas. Barcelona: Viguera, 2012

32. Tirapu-Ustàrroz J, RIM, Maestù Unturbe F. Manual de Neuropsicología 2.? ed. Barcelona: Viguera; 2011.

33. M. Fuster J. The prefrontal cortex. Fifth Edition. USA: Elservier Science: 2015

34. Bombín-González I, Cifuentes-Rodríguez A, Climent-Martínez G, LunaLario P, Cardas-lbáñez J, Tirapu-Ustárroz J, et al. Validez ecológica y entornos multitarea en la evaluación de las funciones ejecutivas. Rev Neurol 2014; 59 (2): $77-87$

35. Ramírez M, Ostrosky F. Flexibilidad cognitiva después de un traumatismo craneoencefálico. Acta de investigación psicológica. 2012; 2 (1):582-91.

36. Toledano A, Álvarez-Adolfo MA, Toledano-Díaz A. Envejecimiento cerebral normal y patológico: continuum fisiopatológico o dualidad de procesos involutivos. An Real Acad Farm (Madrid). 2014; 80, (№ 3): 500-539

37. Cepeda M, Hickman H, Arroyo R, et al. Índice de dificultad en la solución de la tarea Torre de Londres en niños y adultos. Int J Clin Heal Psychol. 2015 1:717-32

38. Wegman T, Sánchez J. Genómica del envejecimiento neurológico Instituto Nacional de Geriatría. (Mex). 2015; 247-56

39. Tirapú -Ustárroz J, García-Molina A, Ríos-Lago M, Ardila A. Neuropsicología de la corteza prefrontal y las funciones ejecutivas. Barcelona: Viguera; 2012

40. García-Molina A. Impacto del daño cerebral adquirido en e funcionamiento ejecutivo. Neuropsicología de la corteza prefrontal y las funciones ejecutivas. Barcelona: Viguera; 2012; 447-60 
Cuadro 1. Relación entre la hipertensión arterial y las funciones cognitivas: Principales alteraciones

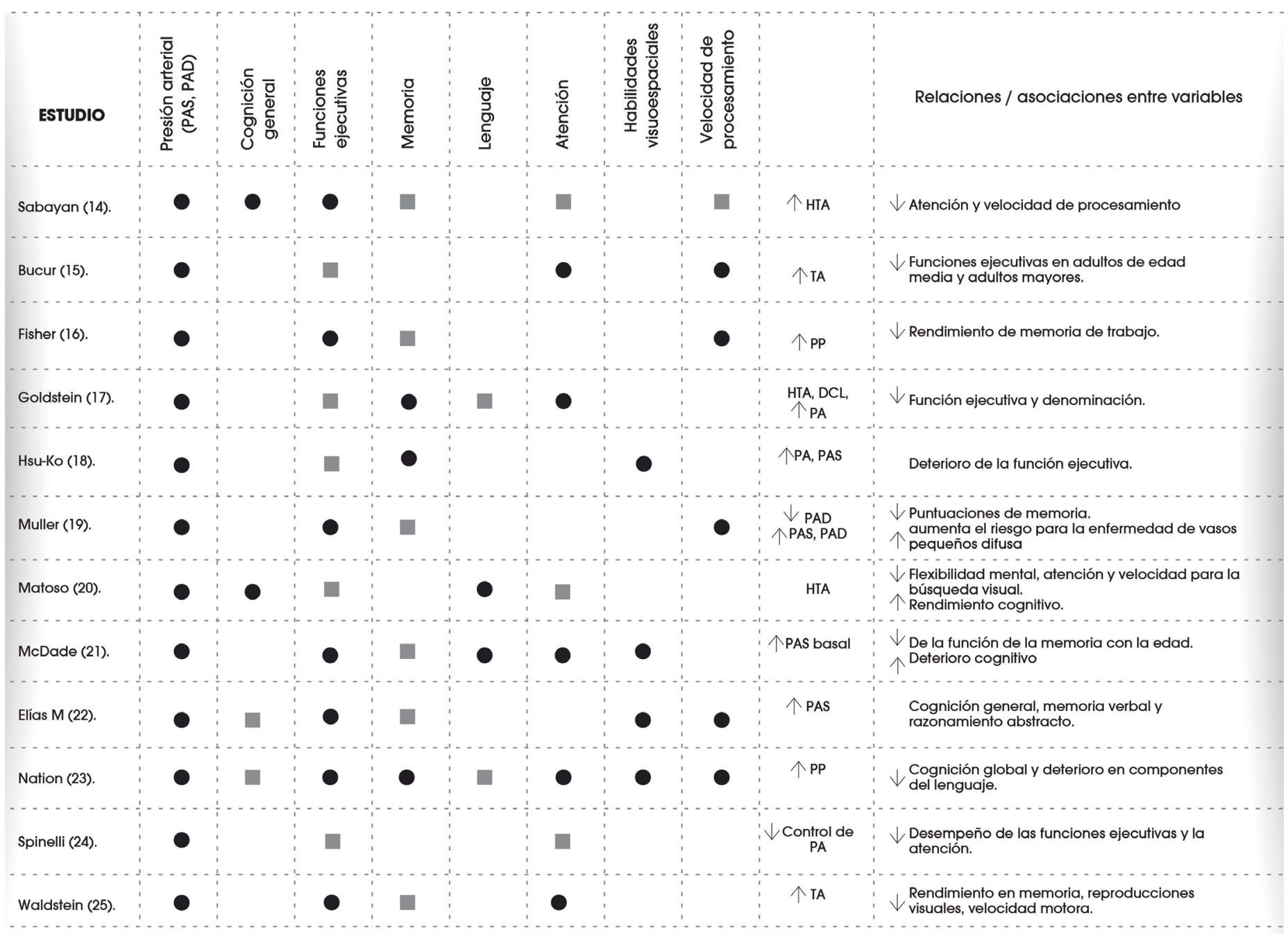

PAS= Presión arterial sistólica, $\mathrm{PAD}=$ Presión arterial diastólica, $\mathrm{HTA}=$ Hipertensión arterial, $\mathrm{DCL}=$ Deterioro cognitivo leve PP= Presión del pulso, $T A=$ Tensión arterial, RR= Riesgo relativo

$\uparrow=$ Aumento $\downarrow=$ Disminución $\bigcirc=$ Variable estudiada $\square=$ Variable estudiada $y$ afectada 